Final Report

August 15, 2000-April 14, 2006

\title{
Metabolic regulation of the plant hormone indole-3-acetic acid
}

\author{
No. DE-FG02-00ER15079 \\ Dr. Jerry D. Cohen Department of Horticultural Science University of Minnesota 1970 Folwell Avenue, \\ 305 Alderman Hall Saint Paul, MN 55108
}

Investigations in vitro of the non-tryptophan auxin biosynthetic pathway.

We studied the environmental and developmental regulation on the use of the tryptophan independent pathway to IAA biosynthesis in several selected systems. These include our demonstration that there is a change in the utilization of the tryptophan pathway during fruit ripening in tomato (Epstein et al. 2002). We showed, using a mass spectrometric/stable isotope method in concert with a tomato ripening-mutant that the conversion from tryptophan dependent to independent pathway was associated with the transition to reduced cell growth that usually accompanies the initial events of ripening. Other studies showed that changes in growth temperature changed the pathway being utilized in Lemna gibba and this apparent environmental effect was further explored by a study of the effect of wounding on the change to tryptophan-dependent IAA biosynthesis (Rapparini et al. 2002). Additional work has looked at this wounding effect by looking for possible differential utilization of the IAA produced following wounding depending on its biochemical origins (Sztein et al. 2002).

A facile radiotracer method for discriminating between the activities of tryptophan-dependent and tryptophan-independent pathways for IAA biosynthesis was developed. This method utilizes the simultaneous exposure of plants to $\left[{ }^{14} \mathrm{C}\right]$ anthranilate in the presence or absence of excess unlabeled tryptophan in order to determine if tryptophan feeding can affect the relative enrichment of the IAA pool. Using this radiotracer method, the activities of the two biosynthetic pathways were analyzed in isolated axes of germinating bean seedlings at various times after cotyledon excision. Unlabeled tryptophan suppressed $\left[{ }^{14} \mathrm{C}\right]$ anthranilate conversion into IAA in isolated axes of different ages immediately following cotyledon excision. On the other hand, tryptophan feeding did not inhibit $\left[{ }^{14} \mathrm{C}\right] \mathrm{IAA}$ accumulation in isolated axes 36 or $120 \mathrm{~h}$ after cotyledon excision. Thus, this method was able to resolve time-dependent differences following cotyledon excision in the biosynthetic activities of the two pathways. Moreover, the present results lend further support to the emerging consensus that the tryptophan-dependent pathway acts to maintain very high IAA levels required for mediating rapid cell proliferation in wounded tissues and, as previously shown, young embryos (Sztein et al. 2002).

The direct conversion of indole to indole-3-acetic acid without tryptophan as an intermediate has previously been shown to occur in vivo, as well as in vitro, with seedlings of plants. In order to facilitate the purification of the enzymes that carry out the enzymatic synthesis of indole-3-acetic acid from labeled 
indole, it was necessary to develop an assay that had both high sensitivity and analytical precision. To obtain the required analytical resolution and to allow definitive product identification, $\left[{ }^{13} \mathrm{C}_{6}\right]$ indole was synthesized for use in GC-MS assays of the enzymatic conversion. Plants have been shown to be able to synthesize indole-3-acetic acid either directly from indole as well as by degradation of tryptophan. Thus, in order to allow the biochemical discrimination between these processes, the synthesized $\left[{ }^{13} \mathrm{C}_{6}\right]$ indole was used as a starting material for a novel enzymatic synthesis of $\left[{ }^{13} \mathrm{C}\right]$ isotopomers of L-tryptophan labeled at specific positions. Together, these isotope-labeled indolic compounds offer powerful new approaches to understanding and differentiating routes of indole-3-acetic acid biosynthesis in vitro and in vivo (Ilic and Cohen, 2004).

\section{IAA biosynthesis from tryptophan.}

In collaboration with Dr. Yunde Zhao, we characterized a dominant activation tagged Arabidopsis line, yucca, which contains elevated levels of free auxin. YUCCA was found to encode a flavin monooxygenase-like enzyme and belongs to a family that includes at least nine other homologous Arabidopsis genes, a subset of which appear to have redundant functions. Results from tryptophan analog feeding experiments and biochemical assays indicate YUCCA catalyzes hydoxylation of the amino group of tryptamine, a rate-limiting step in one of the tryptophan-dependent auxin biosynthesis pathways (Zhao et al. 2001).

A graduate student, Angela Hendrickson, studied the Trp-D IAA biosynthesis in maize endosperm. The tryptophan-dependent pathway is the primary system utilized in maize endosperm during development. This pathway and its intermediates have not been fully elucidated, nor have the enzymes performing the reactions been identified and characterized. We focused our work on development of methodology for optimizing the conversion of stable-labeled tryptophan $\left(\left[{ }^{2} \mathrm{H}_{5}\right] \operatorname{trp}\right)$ to IAA in the maize endosperm system. The amount of synthesized IAA was measured using GC-MS-SIM analysis and this appears necessary to avoid problems of data analysis. This procedure uses $\left[{ }^{13} \mathrm{C}_{6}\right] \mathrm{IAA}$ as an internal standard to determine the amount of $\left[{ }^{2} \mathrm{H}_{5}\right]$ IAA produced. This optimized reaction was also used to determine intermediates in the biosynthetic pathway (such as indoleacetaldehyde) using an interference method we call "cold trapping". This method helped in identifying the enzymes involved in the conversion since it allowed protein purification to proceed along with critical identification of reaction intermediates. Optimization included assessing appropriate cofactors, buffers, and experimental conditions to convert the maximum amount of tryptophan that the system can handle. Most critical was the ability to purify the system using cell fractionation techniques and solubilization of the proteins using specific membrane detergents. Using the isotope-trapping techniques, putative intermediates in all tryptophan-dependent IAA biosynthesis pathways postulated were analyzed and tested for their involvement by examining three factors: 1) if the intermediate was present in endosperm, 2) if the putative intermediate was converted to IAA, and 3) if the putative intermediate could interfere with tryptophan conversion to IAA when fed to endosperm in 20 times excess of tryptophan. No putative intermediates matched all three factors, pointing to a biosynthesis pathway that bypassed traditional intermediates. This was confirmed by analyzing radioactive metabolites of tryptophan in endosperm enzyme extracts. We thus identified a new pathway for IAA biosynthesis which consists of a small protein 8-16 kDa, which covalently binds tryptophan and produces IAA. The bond is most likely a thio-ester, with lability around the chiral center which allows for release of a racemic mixture of tryptophan, although only L-tryptophan is used (Culler, 2007). 


\section{Auxin biosynthesis during zygotic embryogenesis in carrot and other developing plant tissues.}

All plants exhibit the property of cellular totipotency, whereby individual cells can regenerate into an entire organism. However little is known about the underlying mechanisms regulating totipotency. Using a preparative microtechnique, we reported an 80 -fold surge in the concentration of free auxin that is correlated with the initial stages of zygotic embryogenesis in carrots. The free IAA concentration increased from a basal level of ca. $25 \mathrm{ng} / \mathrm{g} \mathrm{FW}$ in unfertilized ovules, to ca. 2,000 ng/g FW in the late globular and early heart stages, then back to the basal level in the torpedo stage. This initial increase in IAA levels is diagnostic of the activity of the tryptophan-mediated pathway for IAA biosynthesis, while the maintenance of the basal levels is attributed to the tryptophan $\neg$ independent pathway for IAA biosynthesis. Our observations are consistent with the hypothesis that the sequential activation of alternative IAA biosynthetic pathways is a critical mechanism for regulating carrot embryogenesis and other instances of plant totipotency (Ribnicky et al. 2002).

\section{Tools necessary to determine the roles of IAA-amino acid conjugates in plants.}

The gene for a specific IAA-asp hydrolase from Enterobacter agglomerans, iaaspH, is a potentially useful tool for modification of IAA homeostasis in higher plants that use the IAA-asp oxidation pathway for auxin catabolism. In order to optimize the utility of this gene for plant modification and to increase the success of obtaining iaaspH transformed plants from culture, we have investigated aspects of IAA-asp hydrolase catalysis. The catalytic characteristics of the IAA-asp hydrolase from Enterobacter agglomerans was studied using ten compounds that are structural analogues of IAA-asp. These compounds were tested as potential IAA-asp hydrolase substrates as well as for inhibition of IAA-asp hydrolysis. Among them, $\mathrm{N}$-carbobenzyloxy-D-aspartic acid (N-CBZ-D-asp) and N-CBZ-L-asp were found to be the strongest inhibitors with more than $80 \%$ inhibition of IAA-asp hydrolysis. Aspartyl-L-aspartic acid and a asp-ser-asp-pro-arg peptide also showed strong inhibitory activities, reducing rates of IAA-L-asp hydrolysis, when added at equal molar amounts relative to the substrate, by $60 \%$ and $65 \%$, respectively. N-CBZ-D-asp was chosen for further kinetic studies and for studies of its toxicity in relation to seed germination because it was a strong inhibitor, exhibited a very low rate of hydrolysis by the IAA-asp hydrolase and was commercially availability. N-CBZ-D-asp was shown to be a competitive inhibitor for the Enterobacter agglomerans IAA-asp hydrolase. Studies of tomato seed germination showed that $\mathrm{N}$-CBZ-D-asp did not affect the rate of seed germination at up to $1 \mathrm{mM}$, but the growth rate of seedlings was significantly reduced when the concentration in the medium was $0.5 \mathrm{mM}$ and higher. These results indicate that, at suitable concentrations, N-CBZ-D-asp should be a useful tool for control of low level expression of the iaaspH in transgenic plants during critical stages of plant regeneration from culture (Chou et al. 2002).

The indole-3-acetyl-L-aspartic acid (IAA-Asp) hydrolase of Enterobacter agglomerans has also shown very high substrate specificity compared to similar IAA-amino acid hydrolase enzymes (ILR1, IAR ${ }_{i} \mathrm{~K}$ ) found in Arabidopsis. In addition, the IAA-Asp hydrolase also exhibits, relative to the Arabidopsis-derived enzymes, a very high $V_{\max }$ (fast reaction rate) and a higher $\mathrm{K}_{\mathrm{m}}$ (lower substrate affinity). These two characteristics indicate there are fundamental differences in the catalytic activity between this bacterial enzyme and the Arabidopsis enzymes. Understanding the basis for these differences may provide a route to optimize the bacterial enzyme to retain the high $\mathrm{V}_{\max }$ but obtain a lower $\mathrm{Km}$. By employing a computer modeling approach, a catalytic residue, His385, from a non-sequence related zinc-dependent exopeptidase (carboxypeptidase G2, 1 $\mathrm{cg} 2$ ) of Pseudomonas was found to structurally match the His405 of IAA-Asp 
hydrolase. The His405 residue is conserved in all related sequences of bacteria and Arabidopsis. Point mutation of this His405 to seven different amino acids (Asp, Glu, Val, Thr, Ser, Cys, or Lys) resulted in completely eliminating enzyme activity. However, point mutation on the neighboring His404 to eight other residues (Arg, Lys, Phe, Tyr, Val, Asp or Glu) resulted in reduction, to various degrees, of the enzyme activity. Amino acid substitutions for His404 also showed this residue influenced the minor activity of the IAA-Asp hydrolase for the substrates IAA-Gly, IAA-Ala, IAA-Ser, IAA-Glu, and IAA-Asn. These results show the value of structural modeling for predicting target residues for further study and for directing bioengineering of enzyme structure and function. Bacterial indole-3-acetyl-L-aspartic acid (IAA-Asp) hydrolase has shown very high substrate specificity compared with similar IAA-amino acid hydrolase enzymes found in Arabidopsis thaliana. The IAA-Asp hydrolase also exhibits, relative to the Arabidopsis thaliana-derived enzymes, a very high Vmax (fast reaction rate) and a higher Km (lower substrate affinity). These two characteristics indicate that there are fundamental differences in the catalytic activity between this bacterial enzyme and the Arabidopsis enzymes. By employing a computer simulation approach, a catalytic residue, His-385, from a non-sequence-related zinc-dependent exopeptidase of Pseudomonas was found to structurally match His-405 of IAA-Asp hydrolase. The His-405 residue is conserved in all related sequences of bacteria and Arabidopsis. Point mutation experiments of this His-405 to seven different amino acids resulted in complete elimination of enzyme activity. However, point mutation on the neighboring His-404 to eight other residues resulted in reduction, to various degrees, of enzyme activity. Amino acid substitutions for His-404 also showed that this residue influenced the minor activity of the IAA-Asp hydrolase for the substrates IAA-Gly, IAA-Ala, IAA-Ser, IAA-Glu and IAA-Asn. These results show the value and potential of structural modeling for predicting target residues for further study and for directing bioengineering of enzyme structure and function (Chou et al. 2004).

Esterification of indole-3-acetic acid (IAA) is thought to be an important component in the homeostatic regulation of the levels of this phytohormone. To better understand the role of the initial step in IAA esterification in the control of IAA levels, transgenic tomato plants were generated that either express maize IAGLU or have reduced levels of the enzyme IAA-glucose synthetase. These plants were obtained by expressing maize IAGLU in either sense or antisense orientation using the CaMV35S promoter. The maize IAGLU probe hybridized to two transcripts $(1.3 \mathrm{~kb}$ and $2.5 \mathrm{~kb})$ in wild-type tomato vegetative tissue and green fruit. The sense and antisense transformants exhibited distinct phenotypic characteristics. Sense transformants showed an almost complete lack of root initiation and development. Antisense transgenic plants, on the other hand, had unusually well developed root systems at early stages in development, and the amount of the endogenous $75 \mathrm{kDa}$ IAGLU protein was reduced. IAGLU antisense plants also had reduced levels of IAA-glucose and lower esterified IAA (Iyer et al. 2005).

Overexpression of the IAGLU gene from maize (ZmIAAGLU) in Arabidopsis thaliana, under the control of the CaMV 35S promoter, inhibited root but not hypocotyl growth of seedlings in four different transgenic lines. Although hypocotyl growth of seedlings and inflorescence growth of mature plants was not affected, the leaves of mature plants were smaller and more curled as compared to wild-type and empty vector transformed plants. The rosette diameter in transgenic lines with higher ZmIAGLU expression was also smaller compared to the wild type. Free indole-3-acetic acid (IAA) levels in the transgenic plants were comparable to the wild type, even though a decrease in free IAA levels might be expected from overexpression of an IAA-conjugate-forming enzyme. IAA-glucose levels, however, were increased in transgenic lines compared to the wild type, indicating that the ZmIAGLU gene product is active in these plants. In addition, three different 35SZmIAGLU lines showed less inhibition of root growth when cultivated on increasing concentrations of IAA but not indole-3-butyric acid (IBA) and 2,4-dichlorophenoxyacetic acid (2,4-D). Feeding IAA to transgenic lines resulted in increased 
IAA-glucose synthesis, whereas the levels of IAA-aspartate and IAA-glutamine formed were reduced compared to the wild type. Our results show that IAA homeostasis can be altered by heterologous overexpression of a conjugate-forming gene from maize (Ludwig-Müller et al. 2005).

\section{Determine using short term in vivo labeling studies in Lemna gibba how tryptophan dependent and independent pathways change based on environmental signals.}

Auxins control many different aspects of plant growth and development and it has been known for over a century that auxin act as the messenger linking plant development to specific environmental changes. An often-overlooked aspect of how auxin accomplishes this involves how the environment alters the metabolism the major plant auxin, indole-3-acetic acid (IAA). We reported that one environmental variable, growth temperature, significantly alters several aspects of IAA metabolism. The model system used was an inbred line of the aquatic monocot Lemna gibba G-3, 3F7-11, grown at temperatures ranging from 5 to $35^{\circ} \mathrm{C}$. IAA levels, the rate of IAA turnover, and the patterns of label incorporation from IAA precursors were measured using stable isotope-mass spectrometric techniques and evaluated relative to growth at the experimental temperatures. IAA levels exhibited unusually high variability in plants grown at $15^{\circ} \mathrm{C}$ and $20^{\circ} \mathrm{C}$. Turnover rates were quite rapid throughout the range of experimental temperatures except at $25^{\circ} \mathrm{C}$, where IAA turnover was notably slower. These results suggested that a transition occurred over these temperatures for some aspect of IAA metabolism. Analysis of $\left[{ }^{15} \mathrm{~N}\right]$ anthranilate and $\left[{ }^{2} \mathrm{H}_{5}\right]$ tryptophan incorporation into IAA showed that tryptophan dependent biosynthesis predominated at $15^{\circ} \mathrm{C}$ however, tryptophan-independent biosynthesis of IAA was the major route to IAA at $30^{\circ} \mathrm{C}$. The effects of growth temperature on auxin levels have been reported previously, however, no prior studies correlated these effects with which pathway becomes the primary one for IAA production (Rapparini et al. 2002).

\section{Auxin response factors ARF6 and ARF8 promote jasmonic acid production and flower maturation}

Pollination in flowering plants requires that anthers release pollen when the gynoecium is competent to support fertilization. We show that in Arabidopsis thaliana, two paralogous auxin response transcription factors, ARF6 and ARF8, regulate both stamen and gynoecium maturation. arf6 arf8 double-null mutant flowers arrested as infertile closed buds with short petals, short stamen filaments, undehisced anthers that did not release pollen and immature gynoecia. Numerous developmentally regulated genes failed to be induced. ARF6 and ARF8 thus coordinate the transition from immature to mature fertile flowers. Jasmonic acid (JA) measurements and JA feeding experiments showed that decreased jasmonate production caused the block in pollen release, but not the gynoecium arrest. The double mutant had altered auxin responsive gene expression. However, whole flower auxin levels did not change during flower maturation, suggesting that auxin might regulate flower maturation only under specific environmental conditions, or in localized organs or tissues of flowers. arf6 and arf8 single mutants and sesquimutants (homozygous for one mutation and heterozygous for the other) had delayed stamen development and decreased fecundity, indicating that ARF6 and ARF8 gene dosage affects timing of flower maturation quantitatively (Nagpal et al. 2005).

\section{Publications:}

Sztein A.E., Cohen J.D. and Cooke T.J. Evolutionary patterns in the auxin metabolism of green plants. Int. J. Plant Sci. 161:849-859 (2000)

Zhao Y., Christensen S.K., Fankhauser C., Cashman J.R., Cohen J.D., Weigel D. and Chory J. A role for 
flavin monooxygenase-like enzymes in auxin biosynthesis. Science 291:306-309 (2001)

Ribnicky, D.M., Cohen, J.D., Hu, W.-S. and Cooke, T.J. An auxin surge following fertilization in carrot: Its significance for plant totipotency. Planta 214: 505-509 (2002).

Rapparini F, Tam YY, Cohen JD and Slovin JP IAA metabolism in Lemna gibba undergoes dynamic changes in response to growth temperature. Plant Physiology 128:1410-1416 (2002)

Ludwig-Müller J and Cohen JD Identification and quantification of three active auxins in different tissues of Tropaeolum majus. Physiologia Plantarum 115:320-329 (2002)

Cooke TJ, Poli DB, Sztein AE and Cohen JD Evolutionary patterns in auxin action. Plant Mol Biol 49:319-38 (2002)

Ljung K, Hull AK, Kowalczyk M, Marchant A, Celenza J, Cohen JD, Sandberg G. Biosynthesis, conjugation, catabolism and homeostasis of indole-3-acetic acid in Arabidopsis thaliana. Plant Mol Biol. 49:249-72. (2002) [Corrected for publisher's errors and republished in Plant Mol Biol. 50:309-32 (2002)]

Chou J-C, Mulbry WW and Cohen JD N-Carbobenzyloxy-D-aspartic acid as a competitive inhibitor of indole-3-acetyl-L-aspartic acid hydrolase of Enterobacter agglomerans. Plant Growth Regulation 37:241-248 (2002)

Sztein AE, Ilif N, Cohen JD and Cooke TJ Indole-3-acetic acid biosynthesis in isolated axes from germinating bean seeds: The effect of wounding on the biosynthetic pathway. Plant Growth Regulation 136:201-207 (2002)

Epstein E, Cohen JD and Slovin JP The biosynthetic pathway for indole-3-acetic acid changes during tomato fruit development. Plant Growth Regulation 38:15-20 (2002)

Cohen JD and Slovin JP Recent research advances concerning indole-3-acetic acid metabolism. In: Mechanism of Action of Plant Hormones, T Bisseling and J Schell, eds. Springer, Berlin (in press)

Cohen JD, Slovin JP and Hendirckson AM Two genetically discrete pathways convert tryptophan to auxin: more redundancy in auxin biosynthesis. Trends in Plant Science 8:197-199 (2003)

Cooke TJ, Poli DB, Cohen JD Did auxin play a crucial role in the evolution of novel body plans during the Late Silurian-Early Devonian radiation of land plants? In: The Evolution of Plant Physiology: From Whole Plants to Ecosystems. AR Hemsley and I Poole, eds. Linnean Society of London and Elsevier Academic Press, Amsterdam pp. 85-107 (2004)

Chou JC, Welch WH, Cohen JD. His-404 and His-405 are essential for enzyme catalytic activities of a bacterial indole-3-acetyl-L-aspartic acid hydrolase. Plant Cell Physiol. 45:1335-1341 (2004)

Ilic N, Cohen JD Synthesis of $\left[{ }^{13} \mathrm{C}\right]$-isotopomers of indole and tryptophan for use in the analysis of indole-3-acetic acid biosynthesis. J Labelled Compd Radiopharm 47: 635-646 (2004)

Normanly J, Slovin JP, Cohen JD Auxin biosynthesis and metabolism. In: Plant Hormones: Biosynthess, Signal Transduction, Action! ( $3^{\text {rd }}$ edition). P.J. Davies, ed. Kluwer Academic Publ., Dordrecht, pp. 36-62 (2005)

Nagpal P, Ellis CM, Weber H, Ploense SE, Barkawi LS, Guilfoyle TJ, Hagen G, Alonso JM, Cohen JD, Farmer EE, Ecker JR, Reed JW Auxin response factors ARF6 and ARF8 promote jasmonic acid production and flower maturation. Development 132:4107-4118 (2005)

Iyer M., Slovin J.P., Epstein E. and Cohen J.D. Transgenic tomato plants with a modified ability to

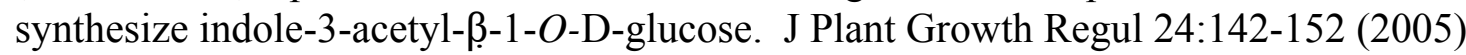

Ludwig-Müller J, Walz A, Slovin JP, Epstein E, Cohen JD, Dong W, Town CD Overexpression of maize IAGLU in Arabidopsis thaliana alters plant growth and sensitivity to IAA but not IBA and 2,4-D. J Plant Growth Regul 24:127-141 (2005)

Culler, AH Analysis of the tryptophan-dependent indole-3-acetic acid biosynthesis pathway in maize endosperm. Ph.D. Dissertation, University of Minnesota, 216 pp (2007) 


\section{Abstracts presented:}

Zhao W, Long JC, Rashotte AM, Ilic N, Cohen JD, Muday GK, Huber SC 2000. Gravity- and auxin-induced changes in invertase gene expression and enzyme activity (Abstract \#511). Final Program, Amer Soc Plant Bio. pp 112

Cohen JD, Cooke TJ, Chou JC, Epstein E, Ilic N, Ludwig-Mueller J, Park S, Slovin JP, Sztein AE, and Walz A. Auxin metabolism: why is it so complex? XXIII Reunion Argentina de Fisiologia Vegetal, Rio Cuarto Argentina, November 2000, Proceedings abstracts, p. 9

Cohen JD, Cooke TJ, Chou JC, Epstein E, Ilic N, Ludwig-Mueller J, Park S, Slovin JP, Sztein AE, and Walz A. Studies on the regulatory biochemistry of auxins. EMBO Workshop on Auxins, Calcatoggio, Cosica France, May 2000 Proceeding abstract T-3.3, p. 21

Cooke TJ, Sztein AE, Poli DB and Cohen JD Evolutionary trends in auxin regulation and their significance for plant embryogenesis. EMBO Workshop on Auxins, Calcatoggio, Cosica France, May 2000 Proceeding abstract T-7.4, p. 32

Cohen JD Alteration of auxin regulation and its potential for the control of fruit ripening. Western Regional Meeting, Canadian Society of Plant Physiologists, Edmonton, AB Canada, May 2000, Proceeding abstracts, p 1 .

Fellner M, Van Volkenburgh E, Cocke A, Ford ED, Cohen JD 2001 Reduced growth responses to red and far-red light in corn plants are associated with ability to maintain productivity in dense plantings (Abstract \#309). Final Program, Amer Soc Plant Bio. pp 79

Chou J-C, Cohen JD, Mulbry WW 2001 Carbobenzyloxy-D-aspartic acid as a competative inhibitor of indole-3-acetyl-L-aspartic acid hydrolase of Enterobacter agglomerans (Abstract \#325). Final Program, Amer Soc Plant Bio. pp 82

Cohen JD 2001 Auxin complexity: pathways, proteins and genes. Abstracts, $17^{\text {th }}$ International Conference on Plant Growth Substances, pp 60.

Rapparini F, Tam YY, Cohen JD, Slovin JP 2001 The effect of temperature on IAA turnover and biosynthesis (Poster \#250). Abstracts, $17^{\text {th }}$ International Conference on Plant Growth Substances, pp 144.

Chou J-C, Welch WH, Cohen JD, (2002) His ${ }^{404}$ and His ${ }^{405}$ residues are essential for enzyme substrate specificity and catalytic activity, respectively, of the indole-3-acetyl-L-aspartic acid hydrolase from Enterobacter agglomerans (Abstract \#762). Final Program, Amer Soc Plant Bio., p.16 
Wong LY, Englen-Eigles G, Cohen J, Markhart A, Gardner G. (2002) The effect of water stress on 2-phenylethyl glucosinolate levels in Nasturtium officinale (Abstract \#189). Final Program, Amer Soc Plant Bio., p. 67

Hendrickson A, Cohen JD (2002) Analysis of the tryptophan-dependent indole-3-acetic acid biosynthesis pathway in maize endosperm (Abstract \#473). Final Program, Amer Soc Plant Bio, p. 117

Tam YY, Barkawi L, Normanly J, Cohen JD (2002) Genomic approaches to auxin biology (Abstract \#466). Final Program, Amer Soc Plant Bio

Barkawi LS, Tam YY, Cohen JD, Normanly J. (2003) Genomic approaches to auxin biology: a high throughput method for the quantitative analysis of indole-3-acetic acid Abs \# 942 Final Program, Amer Soc Plant Bio

Hendrickson AM, Cohen JD (2003) Analysis of the tryptophan-dependent indole-3-acetic acid biosynthesis pathway in maize endosperm Abs \# 624 Final Program, Amer Soc Plant Bio

Vosberg AR, Cohen JD (2003) Qualitative and quantitative analysis of indole-3-butyric acid conjugates in Arabidopsis thaliana Abs \# 626 Final Program, Amer Soc Plant Bio

Wong LY, Cohen JD, Gardner G, Markhart A (2003) The effect of defense signaling molecules on the content of 2-phenylethyl glucosinolate in Nasturtium officinale R. Br. Abs \# 322 Final Program, Amer Soc Plant Bio

Hendrickson AM, Ludwig-Muller J, Cohen JD (2004) Analysis of the typtophan-dependent indole-3-acetic acid pathway in maize endosperm. 18th International Conference on Plant Growth Substances, September, 2004 Canberra, Australia (Invited talk, AH)

Ye S, Cohen J (2004) Identification and characterization of pyruvate decarboxylase ( $p d c)$ gene family members in Arabidopsis. $17^{\text {th }}$ International Conference on Arabidopsis Research (Abstract 346). June 2006

Barkawi LS, Tam Y-Y, Tillman JA, Calio J, Al-Amier H, Emerick M, Normanly J, Cohen JD (2004) A high-throughput method for the quantitative analysis of indole-3-acetic acid and other auxins ASPB meeting (Abs \# P45012), July 2006 